\title{
Ergonomics and Ergonomic Analysis of Driving Education System by Personal Car-2
}

\author{
Kata Car ${ }^{1}$, Julijana Hadjina ${ }^{2}$, Mirela $\mathrm{Car}^{3}$, Domagoj $\mathrm{Car}^{4}$, Miroslav $\mathrm{Car}^{5, *}$ \\ 1 Phoenix Zagreb, \\ Republic of Croatia \\ ${ }^{2}$ West Health Center, \\ Outpatient Clinic Zagreb, \\ Republic of Croatia \\ ${ }^{3}$ Phoenix Zagreb, \\ Republic of Croatia \\ ${ }^{4}$ Phoenix Zagreb, \\ Republic of Croatia \\ ${ }^{5}$ Faculty of mechanical engineering \\ and naval architecture, Zagreb \\ University of Zagreb \\ Republic of Croatia \\ ${ }^{*}$ Corresponding author's email: car.miroslav [AT] gmail.com
}

\begin{abstract}
ABTRACT---- The subject of IT-supported car training/education system was selected for the research below in the previous article. For the well-known hypothesis, out of the three existing parts, the driver-machine-car subsystem was first selected to handle the theoretical hypothesis; presented later. Thus, the following eight subsystems or parts for human participants are listed: nerve area; sense of sight or visual organ of the eye; hearing aid or hearing organ ear; motion observations; psychological factors, mental and psychomotor properties; individuals' reactions and causes of reactions; types of drivers; the influence of alcohol on road safety. In the practical part of the article, based on the vast amount of data, several analyzes were conducted. The vast amount of data requires multiple publication of the complete content of the thesis, where each new content unit / article must have both parts, both theoretically and practically. To our knowledge, a unique list of 19 types of errors was made, with a detailed description of them. The terms of different types of driving are explained in particular: polygon, city traffic and examinations, as well as the differences between them. In the series of analysis, the terms driving type, teaching unit, candidate and driving hours were taken as the independent variable, while the error types with the predominant frequency characteristic were taken as the dependent variable. Practical parts are supported with IT processing. Appropriate ideas and measures for improvement have been proposed. The article gratefully acknowledges Prof. B.Sc. Marijan Biščanić and prof. Ph.D. Dragutin Mikšić for their contribution.
\end{abstract}

Keywords--- ergonomics, education system, driving frequency of errors, IT processing

\section{INTRODUCTION}

One of the basic processes contains the following: a person/candidate - driver perceives signals from his/her personal car and environment (others: vehicles, persons, traffic signs, etc.), processes and processes data at certain times and produces deciding what action to take. The action achieved by acting on the control devices/controls of a car, which are in operation (press and release the clutch pedal/clutch, pressing and releasing the brake pedal, pressing and releasing the accelerator pedal/acceleration or deceleration, shift lever changed her/transmission, etc.), and by signaling to other road users (switching turn signals on and off, switching on lights, etc.). The following eight subsystems or parts for human participants are listed and described for the system: nerve area; sense of sight or visual organ of the eye; hearing aid or hearing organ ear; motion observations; psychological factors, mental and psychomotoric properties; individuals' reactions and causes of reactions; types of drivers; the influence of alcohol on road safety.

In the practical part of the article, based on the vast amount of data, several analyzes were conducted. To our knowledge, a unique list of 19 types of errors was made, with a detailed description of them. The concepts of different types of rides are specifically explained: polygon, city traffic and examinations, as well as the differences between them. In 
the series of analysis, the terms of driving type, unit, candidate and driving hours were taken as the independent variable, while the error types with the predominant frequency / frequency characteristic were taken as the dependent variable. Practical parts are supported by IT processing. Appropriate ideas and measures for improvement have been proposed. The articles are a show of gratitude to the late B.Sc.Mech.Eng. Marijan Biščanić and prof. Ph.D. Dragutin Mikšić for their contribution.

\section{THEORETICAL SETTING OF ITEMS THE HUMAN-MACHINE/PERSONAL CAR IN DRIVING EDUCATION}

Subsystem driver-MACHINE/passenger car contains in training driving machine/car three important factors: (1) man/candidate-men and women or driver partners, (2) human/instructor, (3) Machine/personal car. In this article, the first human participant describes the first subsystem (1) man/candidate or driver partners, are not described, and does not participate in the analysis, other human participant, (2) human/instructor, while the third participant, (3) machine/passenger car, canines to be included and described. The most important influencing factors for a man / candidate - a driver - are the main elements of psychology: pediatric, ergonomic, evolutionary, cognitive, organizational, positive, traffic, personal, consumer, sports, developmental, social, school, health, while for the machine/ passenger car, those are technical characteristics, with the characteristics below, [14, 15, 20, 21, 22].

A man / candidate or driver. The most important parts of said participant are: nerve / nerve area; a sense of sight or visual organ of the eye; the sense of hearing or the auditory organ of the ear; motion observations; psychological factors, mental and psychomotor properties; individuals' reactions and the causes of those reactions; types of drivers; the impact of alcohol on road safety. An important summary of the contents of each section is presented as follows:

a) Nerve/nerve area. Contains: (1) Functions in the nervous sub-system. Nerve system has three basic functions: it regulates the work of individual organs of the human body; coordinates the work of organs for the human body; organizes the work of the human organism with its environment and forms the basis for the entire psychic life of man; (2) major parts of the nerve area. Area nerve is very complex, and consists of the structure of neurons that make specific organization or parts of nerve areas. The main components are: a peripheral part and a central part. The peripheral part consists of neurons and parts of neurons lying outside the cranial cavity and spinal cord. It is made up of neurons in the senses and neurons located between the senses and the spinal cord. It also includes neurons and nerve fibers of some motor and sensory neurons, whose nerve cells are located in the brain and in the spinal cord, in the form of groups or ganglia. The central part is further divided into the spinal cord and the brain that is in the cavity of the spine (the inner part is nerve cells, so the specified one is gray, while the outer part is nerve fibers, so it is white). Two parts are important in the midbrain, the thalamus and the hypothalamus. Nerve impulses are transmitted by neurons to the smooth muscles of the abdominal organs and glands, or to other organs and parts of the body, so the division of the nervous system into autonomic or vegetative and somatic nervous systems can be performed, according to this criterion. Autonomous or vegetative nervous system with the congestion of two parts: the first part, part of the sympathetic (sympathetic nervous system) leads to increased heart rate, increased the blood pressure, pupil dilation, and to other changes in the organs of the body. This means that it is the answer for stimulating response-related activities in a "fight or flight" model; the second part, the parasympathetic part (parasympathicus), affects the functioning of individual organs when the person is in a calm and unsettled state and acts in the opposite direction from the sympathetic part (calms the heart, reduces blood pressure, etc.). This system is therefore ready to respond according to the "rest and work metabolism" model. The somatic nervous system is not the subject of interest in this article , [3, 6, 11, 14];

b) the sense of sight or the visual organ of the eye. The visual organ of the eye

accomplishes its function through its following features: retinal adaptation; pupil diameter; visual acuity; point of view (the point of best vision by day is the so-called "yellow spot." At night, therefore, it must be viewed centrally/extra-verbally at the light, not through the central unit. The three basic subfunctions of the eye would be: (1) sensitivity to light differences and shadowing, ( 2) color vision and for color blindness. Light is electromagnetic phenomena in the form of waves. The eye receives waves of wavelength from 380 to 280, and these waves are of corresponding lengths, and in these limits it gives the experience of light and color; (3) sense of sight, the eye, in the traffic. The candidate/men and women or driver to the traffic had to meet the requirements with the following characteristics: the width of the field of vision, the ability to assess (physical) distance, adaptation and accommodation of the eye. There is a number of situations in traffic caused by the organ of sight, the eye (visual field constriction etc.). Therefore, in the phase of training drivers, before issuing a driver's license, implement and test the eyes, as the most important senses of sight. Reaction time of vision is the time between the moment of light stimulation of the senses and the moment of reaction of the senses. It is large and provides visual control of automated movements (some movements are shorter than 1/10 s); (4) established values of eye reaction. For the analysis of the traversed path in the reaction time, it is necessary to know the essential measurement quantities and the associated measurement units for decision, which are the path and time of the reaction. Traffic events and situations require several important time magnitudes and data, such as: time taken to perceive the situation from the perspective of the eye, in order to obtain an impression of the degree of danger, that is, the need or desire to take action, the time required for changing the angle of observation of the eye by light changes it may be especially important in the sum of the temporal elements; the 
eye time required to adjust the eye to light changes can be very interesting, because of the conditions that the road with the environment and the behavior of road users may impose.

By rotating the eye from left to right and/or the head, relative to the symmetry of the eye and / or the head, for different angles of rotation of the eye and / or the head, the field of vision is shown in Figure 1. The time required to change the angle of observation of the eye in the sum of the time elements may be especially important. Laboratory and practical results show: at rest, the field of vision is up to 140 degrees, or +/- 70 degrees for left-to-right rotation of eye symmetry; when rotating at a speed of about $25 \mathrm{~km} / \mathrm{h}$, the field of view is up to 100 degrees, or $+/-50$ degrees for turning left-right from the symmetry of the eye; when rotating at about $150 \mathrm{~km} / \mathrm{h}$, the field of vision is up to 40 degrees, or $+/-20$ degrees for left-to-right rotation of eye symmetry, [7,12 16, 24];

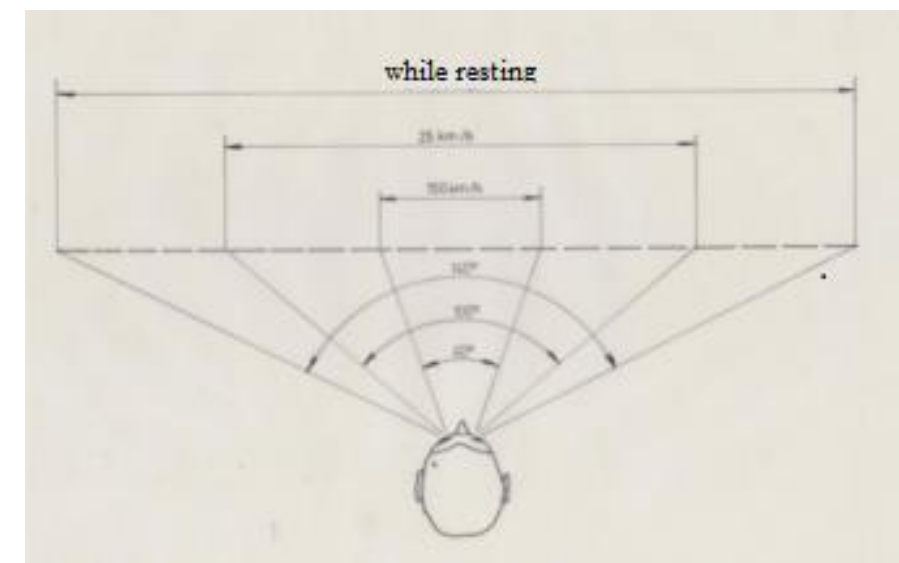

Figure 1. Human field of vision, depending on several factors Source: Biščanić, M.: Ergonomic Features in the Training of Driving a Car, Graduate Thesis at the Faculty of Mechanical Engineering and Naval Architecture - Zagreb, University of Zagreb, Zagreb, 1975, Republic of Croatia

c) hearing sense or hearing organ, the ear. Hearing plays a large role in communication (communication, speech development, musical creativity, etc.) and it is a very rich source of useful information regarding the nature and location of the objects and phenomena that surround us. During the auditory experience, you need: (a) an auditory source, (b) an elastic medium for the source, (c) an auditory organ, and (d) a nerve / nerve center. Sound waves are longitudinal vibrations of sound particles, which are represented by a sinusoid wave. Human sensitivity to effects receiving is highly developed and ranges from 16 to $23000 \mathrm{~Hz}(23 \mathrm{kHz})$, ranging from 10 to 11 octaves of tone scale. The highest sensitivity is in the range of $2000 \mathrm{~Hz}(2 \mathrm{kHz})$ to $3000 \mathrm{~Hz}(3 \mathrm{kHz})$. Air vibrations below $16 \mathrm{~Hz}$ are considered to be infrasounds, and those above $23000 \mathrm{~Hz}(23 \mathrm{kHz})$ are considered to be ultrasounds. Ear in traffic: according to statistics, oscillatory information, there is no research on the participation of deaf people in traffic. Based on the small number of deaf drivers, it can be concluded that the use of sound signals does not provide traffic safety, and vice versa [10, 23];

d) movement observations. It is important to determine the positions in the individual motion observations. One is able to determine the direction, size and speed of movement of parts of the body to move, in the following situations: (1) actively, when stimulated by ourselves, (2) passive, when stimulated by others. There are several groups of information in the area of this sensitivity, namely: (a) kinesthetic information, motion related performance, (b) statosthetic information, positioning/resting, (c) dynamic information, indicating movement strength, (d ) oscillatory information, refers to flicker.

In psychology, motion observations were once thought to be information from other senses. However, it has been proven to be special senses and sensitivities, compounded by muscle and tendon sensitivity, joint sensitivity and bone sensitivity. The physiologists have shown that muscle and tendon sensitivity are still present [1, Charles Bell, $1833 ; 1774-.1842$, Scotland], intersecting movement wires in appropriate parts of the body. Organs and sensitivities include helixes of nerve endings wrapped around muscle spindles, sensitive to muscle contractions (stretching and tightening), bundles of nerve/nerve fibers in connective tissue and nerve/nerve spindles with tendon. It can be concluded that stimuli do not reach consciousness, but remain at the level of reflexes. In addition to this, there is information about statics and dynamics, that is, information about the position of the head and body with respect to the force of gravity and the vertical. The feelings here arise at the beginning and at the end of the rotational movement and at the acceleration or deceleration of the movement, $[1,2,8$,$] .$

e) psychological factors, mental and the psychomotor properties.

Psychological Factors / mental activities are understood as human ideal activities. Activity 
cross-orientation (a frequent activity of the driver's work activity) includes the following sub-activities: turning the head, turning the eyes, concentrating, altering the stimuli and braking processes in different parts of the cerebral cortex, applying visual purple (important first light-sensitive rhodopsin pigment or visual purple, one of the four types of molecular protein opsin) in the retina of the eye, and perceived understanding of a number of other physical, physiological, biochemical and psychological features. According to the main areas of psychological life, with properties are divided in 3 groups: perception (observation), practical/psychomotor skills and intellectual abilities.

(1) perception : mental process a complete entrainment of individuals and relations between them. Our knowledge, activity or behavior depends to a great extent on our perception. Perception provides information about everything in the environment, and again, it affects our activities and allows us to adapt to the environment. In the study of perception, most attention is paid to individual sensory activities, but also to various important features within the same senses: sight, hearing, kinesthetic, etc. In the area of the senses, color sensitivity, types of color sensitivity, auditory perception of tones (low , middle and high frequencies), differentiation of auditory stimuli, differentiation of color tones, etc. There are great practical benefits for reliable selection of people for particular jobs in the field of transport. For example, kinesthetic sensitivity, a group of information, is a trait of determining the position and movement of certain parts of the body, except the head, over the sense of sight. That feature is invaluable for drivers. There is also a distance estimation feature, that has been found to be a special feature, which is important for pilots and motorists and is seeking to develop as much as possible. Without it, moving in heavy traffic, parking, garages and the like would not be successful. It is a special feature, because it is a preparation for observation.

(2) psychomotor abilities : they are paramount to the driver. Machines, controls, etc. are controlled based on human psychomotor abilities. Psychomotor skills are dependent on the anatomic-physiological features of individuals. Life needs give birth to desires (goals, motives, impulses) and they create actions (means to achieve the goal). When performing voluntary movements, which are immediately behind the desire, one is aware of the movement. Without desire, the movement would be pointless. With this approach, the cerebral cortex movement centers are called psychomotor centers. When the driver turns the steering wheel, he performs movements that are determined by the stimuli of the outside world and which achieve a conscious goal. An element of a person's psychomotor activity is a psychomotor or moving action, which represents the solution of an elementary task, that is, the achievement of an elementary conscious goal in one or several movements. The psychomotor activity that develops in the exercise process is called the psychomotor habit. There are two groups of psychomotor processes: sensomotor and ideomotor.

The general approach: in the workplace, therefore, and in the driver's seat, there is a

sensory field, or part of the workplace, that is the source of professionally important information. In the sensory field are only those stimuli to which the worker must react, for example, in the sensory field of drivers belong: roads, sidewalks, transportation, traffic lights, valves, engine noise, bumps, etc. There is also a motor / engine field respectively, that part of the workplace where a man acts through his labor movements. The motor field consists of: steering wheel, gear lever, clutch pedal, accelerator pedal, parking brake lever, foot brake pedal, etc. In each work movement of the realized psychomotor process, its three modes are distinguished: mechanical, physiological and psychological. Mechanical and physiological vision cause understanding of psychological vision. Mechanical properties of the working movements are: silence/trajectory (shape, with the direction and volume of labor movements); speed and distance traveled (evenly accelerated, evenly slowed down, unevenly accelerated and unevenly slowed labor movement); the pace or frequency of repetitive cycles of uniform movements; by force of execution, with pressure or train. Psychological analysis shows that the working movement trajectory can be free, stencil or constraints on. The speed of various work movements is in a large range. From the psychological point of view, the optimum, the most comfortable and the maximum speed are distinguished. There are free and dictated speeds and can be imposed by working time deficits. The driver also has a shortage of time, therefore, a higher speed of movement when involved in the traffic, or in the direct avoidance of a traffic accident, it is only important that the working movement is absolutely accurate. Movement tempo can range from 1 to 2 per second (torso) to 10 movements per second (finger taps). Differences in the force of labor movements can be seen, for example, in the comparison of the operation of the carrier (force greater than $100 \mathrm{~kg}$ ) and the man who controls the landing of the aircraft (force about 10 grams). Important are the works devoted to the study of sensory motion corrections or inverse afferents [1, PK Anohin, 1961 and 1973; 1898-1974, Russia], which determine the accuracy and coordination of movements. According to this view, coordination is not a particular accuracy or sharpness of effector/executive nerve impulses, but a special group of physiological mechanisms that allow for continuous organized cyclic interaction between receptor and effector/executive processes. In psychological analysis of working movement, it is important to know the target, which is achieved by a further motion. Different goals can be achieved with the same movement, and different goals can be achieved with the same movement.

Generalized psychological features of the labor movement are split in the following groups: basic, corrective; additional; malign; superfluous; wrong. Hand-to-self movements are more accurate but also slower than "handto-self" movements, where the latter are less accurate but also faster. The dependence of the time norms of categorized work (micro) movements, their combinations and groups on a number of factors is evident in innumerable tables of systems, such as : Predetermined Times System PDTS, for example, Method time measurement MTM, Work Factors WF, down to systems such as the Maynard Operation Sequence Technique MOST, and others.

(a) Sensomotor processes: Relationship between perception and corresponding 
work movement within work activities can be realized in 3 groups/forms: simple sensory responses, complex sensory responses and sensomotor coordination, acoustic and tactile-motor reactions. There are special reactions where the kinematic senses are the sensomotor moment. Special activities exist in many professions and are an indicator of exercises (for pilots in landing planes; for drivers in overtaking and rebuilding vehicles, etc.). Conditional reflexes were found to be the fastest formed, based on auditory stimuli, slower based on visual stimuli, and slowest based on skinmechanical ones. From a psychological point of view, sensomotor reactions may be actions, or they may just enter the structure of psychomotor actions. There are 3 psychological reactions in each sensomotor reaction: the perception reaction (sensory phase of the reaction); more or less complex processes associated with the perceived (central reaction phase); processes that determine the beginning of the action (motor phase of the reaction). From a physiological point of view, the sensomotor reaction is a conditional reflex.

The total reaction time can be represented by the formula

$$
\mathrm{ERT}=\mathrm{LRT}+\mathrm{MRT}, \text { milliseconds ms (1) }
$$

where is:

ERT- the reaction time of the reaction,

LRT-latent reaction time,

MRT- motor reaction time.

Based on the survey of the average LRT of 9 known researchers, it is possible to conclude the following: data from 9 researchers are representative; LRT is tested for three types of sensory stimuli (light, sound, electrical stimulation of the skin); for each type of sensing stimulus differential/range between the maximum and the minimum value is 30 to $50 \mathrm{~ms}$, with a minimum range (the light 150, audio 120, an electric stimulus to the skin $100 \mathrm{~ms}$ ) to the maximum range (light 244 audio 180, skin electrical stimulus $201 \mathrm{~ms}$ ); legality shows related LRT lights> LRT sound> LRT electrical signal to the skin, where the parts of LRT are:

- Run time (nervous processes, perceptual part of vision) 20 to $60 \mathrm{~ms}$,

- the time of nerve signals in the optic nerve is 2 to $3 \mathrm{~ms}$,

- process time in the cerebral cortex 12 to $15 \mathrm{~ms}$,

- rest of the time, the motor part of a simple sensomotor reaction

$$
\text { Total (without time surplus ) from } 34 \text { to } 78 \mathrm{~ms} \text {. }
$$

On the basis of a special study for the dependence of the reaction, it is possible to conclude the following: the previous rule in $(1)$ is the dependence on the type of stimuli (data are representative: LRT was tested for 3 types of sensory stimuli: bright values are 60 to $100 \mathrm{~ms}$; further dependence shows legality LRT sensomotor response (perception)> LRT motor parts (movement), wherein the first part is bigger than the other by about $100 \mathrm{~ms}$. The natural action of the much more common test for sensomotor reactions than the motor ones. LRT values vary depending on different factors and their characteristics: the use of a single finger on the hand, the use of a single color, the use of the peripheral or central brain, the intensity of stimuli, the movement of the vehicle (instant stop of a line or other action ),

(b) Both ideomotor processes: these processes link perception / image

with movement execution. Kinesthetic senses cause involuntary movement, while voluntary movement is the result of thinking about sound, electrical stimuli on the skin; for each type of sensory stimuli difference / range between the maximum and minimum (driver activists) movements because of him/her, provided that the said senses must connect with all the cells of the cerebral cortex. In the narrow sense of the above processes may, among other things, cause the : unintentional mixing of the human/instructor in the work of the candidate; inadequate operation with dual vehicle controls; can cause wrong, instead of corrective, actions. In broad terms listed will not process the role of working movement with the image of it. With the central problem and physiological point of view appearance, there is a pedagogic problem of awareness training in motorcycle/motor habits, as well as the problem of self-control during training. A classic example of psychomotor is to calculate the stopping distance of a vehicle. It is the same part of the vehicle's distance traveled, from the moment the obstacle or danger was detected, until the moment the vehicle stopped.

Figure 2 shows the elements of the vehicle stopping distance. 


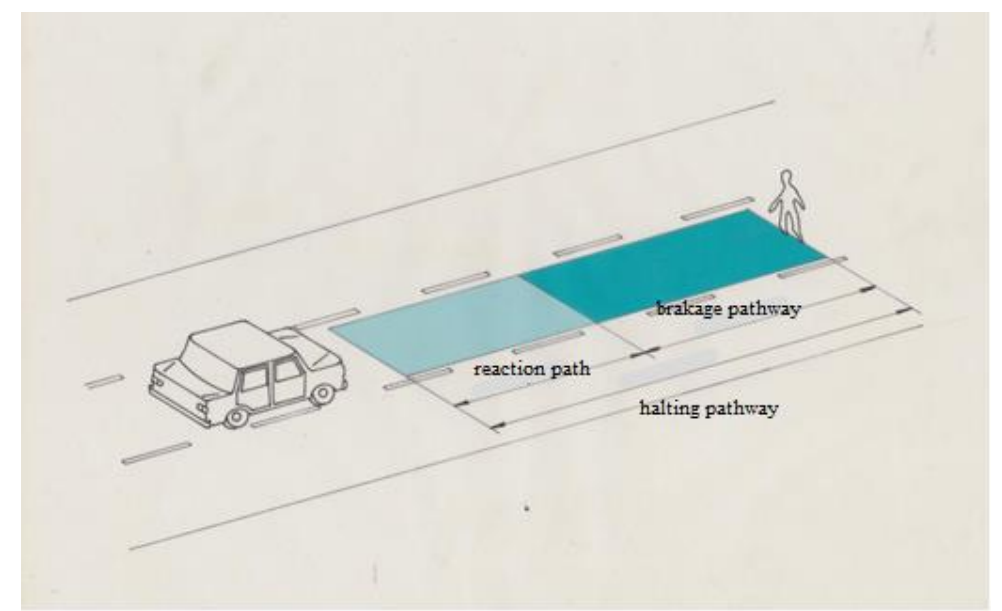

Figure 2. Elements for vehicle braking pathway

Source: Biščanić, M.: Ergonomic Features in the Training of Driving a Car, Graduate Thesis at the Faculty of Mechanical Engineering and Naval Architecture Zagreb, University of Zagreb, Zagreb, 1975, Republic of Croatia

How is the relationship known? Vehicle stop=Response path+Braking distance, meters

Vehicle braking pathway $=$ Braking $/$ Breakage Pathway, it can be concluded that, after deliberation, the vehicle speed and response time increases, or time response and thus increases the braking pathway of the vehicles. Response time depends on several factors: driver alert or not; driver rested or tired, beginner or experienced driver; the driver drives constantly or temporary; driver under the influence of alcohol or other harmful substances or not.

(3) and non- intellectual traits are divided into: (1) memory traits (smaller proportion), and (2) traits opinions (higher proportion). Attribute memory: includes many different and mutual relatively related traits. It is usually divided into: (a) what is remembered (object associations, system, etc.,), and (b) the content to be remembered (figurative, symbolic, schematic, etc.) Reviews attribute, to that trait belong: (a ) knowing and cognition , (b) activating or re-acquiring information, (c) information. Previous traits are divided by: type of subject, cognition, type of content. The productive feature allows new data or notifications to be generated based on known data or information. They are divided into: the feature of convergent thinking (thinking leads to the conventional answer) and the properties of divergent thinking (thinking goes in different directions, looking for something new). The trait of evaluation determines the appropriate value of the knowledge or something new. The relationship between intelligence and driving quality indicates the results of some research in the USA: one must possess average or above average intelligence to be a good driver; if the driver's intelligence increases, the rate of accidents decreases. The basic explanation includes the connection between intelligence and the emergence of new driving conditions or situations (quick and accurate thinking, predicting the behavior of other road users, etc.). The driver of higher intelligence is more successful in coping with new situations than the one with lesser intelligence. Other research shows: higher intelligence drivers are, most often, among the best drivers. Intelligence returnees drivers in traffic accidents is about $20 \%$ smaller than the intelligence average, et al ., [4, 13, 15, 17, 18, 19, 20, 21, 25];

f) the reactions of individuals and the causes of those reactions. To meet a person, you need to know person`s essential motives, but also their behavior (conflicting situations that present frustration), as well as a person's attitude when certain motives cannot be satisfied. There are 3 types of conflicts: (1) conflict due to commitment due to two attractive goals (easily manageable); (2) conflict resulting from the choice of two unpleasant activities (first the choice of a remote situation in an inability to resolve, escape from the situation); (3) conflict in the existence of two opposing motives, where one drives execution and the other avoids execution (most often indecision, with attempt and abandonment). The types of reaction to frustration, regardless of the cause, are: realistic (appropriate) and unrealistic (inadequate). Most of the time, the real-life reaction is the everyday problems in which the goal is often sought in various ways. Negative consequences of frustration are at the same time an obstacle and condition for the development of some positive traits of a person. The most common negative consequences of frustration are: anxiety, aggression, where anxiety and aggression are linearly positively correlated with each other; convenient behavior, which only makes it difficult to deal with. Among the most serious consequences of neuroses belong neuroses from psychosomatic disorder. Frustration tolerance is resilience to frustrating behavior (failure, effort to reduce frustration by easier submission), [6, 26, 27];

g) types of drivers. The term "types of drivers" has been used conditionally, because there is no scientific typology for drivers by some selected criteria. Relevant institutions in the world work in a variety of ways (team and others) with a scientific approach to solving typology and other activities related to the former. In the literature and in 
practice, most often referred to some criteria (driver behavior towards other traffic participants: according to participants in their own vehicle, and according to his own vehicle, according to other participants in a car driving - driver, the vehicle and the participants in the car, its pedestrian/activists traffic, etc., according to which, there are the following types of driver with appropriate characteristics: selfish drivers, men and women; driver that shows off; emotionally unstable driver; temperamental driver; driver that constantly justifies; brute driver; driver with a sense of guilt; irresponsible driver; conscientious driver; careless driver; driver that has repeated traffic accidents; cultured driver. There are other typical behavior of drivers, mainly from the group of mentally unbalanced person (for example, those who constantly "stick to others", those who constantly "jump in front of others", those who constantly drive in the middle of the road ("hedgehogs") and others. A particular problem, which is being investigated more and more extensively, is the problem of identification of the driver with the car. It has been found that not a small number of drivers have been identifying with the car and its characteristics (type, size, speed, color, etc.). It can be generally concluded that the drivers listed above behave significantly differently with the car than they did without it; One of the later surveys from 2014 listed the following types of driver: englishmen, cellphone users, twins, egoists, reservists, trip takers, drowsers, drivers who wait red pointers, [18];

h) the impact of alcohol on road safety. In this article, the subject of study is the influence on participants

in the case. The impact is, however, the largest in the drivers vehicles, thus higher than in other participants. Traffic accident statistics cite a number of causes, such as speeding, carelessness, disrespect, etc., especially interesting for those with fatalities. A man is specifically listed as a "personal cause", and this information is always listed as one of the first in all the classifications. The effects of alcohol can be viewed from three points of view: medical, psychological and legal. The most interesting point of view is legal, since it is actually a legal point of view. Regardless of the variant of the law throughout history, on occasional variations of the dominant alcohol value and the limit value of the alcohol concentration in the blood, the stated value is usually $0.5 \mathrm{ppm}$. Drivers with a higher value than the limit should not participate in traffic as it is not capable of a proper and quality driving of the vehicle, all on the impact of the concentration of alcohol in the driver, according to the following data: first the concentration of 1,0 per mille of alcohol disables about $87 \%$ of drivers for proper driving, and secondly, a concentration of $1.4 \mathrm{ppm}$ virtually disables all motor vehicle drivers (although this is not the upper limit of the generally defined degree of heavy drinking) for proper driving. The reflex movement extends the movement time by 1 second, or so-called. "psychological second," to a greater value; extends past the ground of the LVR, which prolongs the time reactions, and thus, extends the brakage pathway. The influential elements on actions under the process of alcohol are: very fast acting, with dynamics, where immediately at the beginning of consumption it acts with a degree of alcoholism about $20 \%$, in the first hour after consumption it acts with a degree of 50 to $70 \%$, while in 2 hours it acts $100 \%$. This makes the alcohol inhibitory. The reasons for these phenomena are different. Analyzes of the dependence of different outcomes/consequences and a number of variables (days of the week, hours of the day or night, parts of the day or night, etc.) indicate the degree of influence of alcohol, [7, 11, 12] .

\section{RESULTS OF RESEARCH AND PROPOSED IMPROVEMENTS EDUCATION / EDUCATION SYSTEM}

Types of errors during the drive. There are a number of questions about the occurrence of driving errors, such as "How many driving hours does it take for a single candidate to be highly automated?", etc., and it is interesting to measure characteristic types of errors (they also contain individual errors or parts of them that work) candidate/men and women from the initial hours of training. Since the potential of concepts and entities for implementation, taken into account the concept/entity activities/elements of driving techniques, in order to observe the phenomenon of types of errors in them. One of the most important reasons is that in normal driving, listed errors should not exist, or should be at the minimum, and the reason that all actions/elements of techniques should become a habit. It is logical that since the driver should not even think about the above reasons, the motive for research is exactly that it is often the way these elements highly automatize after many hours of driving. A criteria of erroneous embodiment is established: said element/type of error were incorrectly performed if the candidate was previously familiar with the element/type of error in the previous units, but was performing them incorrectly again.

Certain types of errors (types have a division on individual errors) have the following lists and descriptions:

1) incorrectly loosen the clutch pedal. This error occurs most when moving the vehicle and is a common situation. Candidate/men and women must, by constantly slowly and gradually pressing the pedal for "acceleration"/ accelerate or slow down, slowly and gradually release the clutch pedal but at the moment, when it feels that pedal has been "accepted" should stop a bit and then further release it. Failure to do so will cause the vehicle to jump or the engine to shut down. The second and different situation is with very light city driving where, with the accelerator or accelerator pedal pressed appropriately, it is necessary to drive or "slide" on the pedal and clutches, or "dose" the accelerator/ acceleration or deceleration strength, while holding the foot on the pedal by pressing or releasing it;

2) engine starts/stops when starting or stopping: the situation occurs if the clutch pedal is poorly/too quickly released, or the vehicle is moving in the wrong gear;

3) clutch pedal is not tight enough: if this happens, it is inconvenient for the vehicle, because the engine of 
the vehicle is not detached from the gearbox and "shrinking" occurs (gear teeth cannot enter properly but tare / "shrink" one at the other);

4) sudden braking: this situation is more common at the beginning of training until the candidate is able to estimate how long it takes, that is, the amount of foot pressure required on the brake pedal to stop or decelerate the vehicle. The aforementioned mistake can be very dangerous, even fatal, later in city driving;

5) pressing the accelerator pedal abruptly: the vehicle starts abruptly, usually jerking, with the accelerator accelerating/decelerating abruptly or decelerating the vehicle abruptly, usually jerking, with driving intermittent and uncomfortable, followed usually by a sudden release of the accelerate or decelerate pedal. It should be accurately dosed pressure on the accelerator "pedal"/accelerated or slowed down,

6) switch lever changing in the wrong gear: candidate/men and women are still not confident enough and they do not know well enough the scheme of transferring the individual gears driving ("speed" has changed), they do so in good faith, or too fast, so they usually are wrong and shift the lever into the wrong gear;

7) insufficiently sensitive, rough or insufficiently dynamic estimated/fast changing gear or "speed" has changed: this situation is most common when a candidate will not know what is necessary, at all, or first, do so "perfect and only" solution found in changing gear or "speed" changed. The vehicle then usually wanders, drives in to a curb or changes direction; the latter is the most common situation always or very often, because before the bend a vehicle must change gears or change "speed";

8) Driving gear is not adapted to driving speed: driving in which the candidate has not shifted the gear lever to a higher or lower gear in proportion to the current speed, but also a situation where the candidate is driving (very) slowly , with the improper, third gear engaged and/or the fourth gear, or drive (very) fast with the vehicle unsuitable for the first or second level of transmission;

9) incorrect posture in the seat of the vehicle: at the beginning of training candidate/men and women do not know how to take a real position on the seat of the vehicle, but, usually tilt to the right or lean in;

10) lose direction: it is a common mistake of the candidate in the beginning and later in the process. The candidate does not yet know the required turn/turn of the lane/control unit and how it must be "handled" with the steering wheel/control unit. Since this error, unfortunately and unexpectedly, occurs at a later stage of training and can be very dangerous, it requires from the outset a strong intervention of the instructor in order to reduce the impact or eliminate the said error;

11) poor steering/steering device turning, or poor turning: the candidate makes a very bad arc at the steering wheel/steering wheel or enters the outer/right side of the road (the cornerstone of the junction of the road and sidewalk). There is usually a particular problem with controlling the steering wheel/steering device when turning;

12) losing direction when changing gears: an error that occurs very often when driving. The first common situation is one involving the release of the candidate's right hand from the steering wheel, which causes problems to the left hand of the candidate who has not yet been trained in steering the wheel/control unit properly. Another common situation is the leaning of the candidate's left hand on the steering wheel/control unit, which is jerked on that occasion;

13) clutch pedal and accelerator pedal/accelerated or decelerated in a non-synchronized operation: any uneven operation of the left and right leg. Changing gears, it is necessary to align work of the clutch pedal and the "accelerate" pedal/accelerate or decelerate following the next procedure: pedal clutch-release "accelerate" pedal/ accelerate or decelerate, gear shifting or "speed" rolled rod clutch release, clutch pedal release - accelerator/ accelerator pedal pressure/accelerate or decelerate. So the left and right leg have to work in sync, because otherwise there is a jerk and a jump in the vehicle. If you press the clutch pedal, and do not let the "accelerate" pedal/accelerate or slow down, you hear a strong sound of the engine, the consequence of which is, most often, suddenly released state of the accelerator and vehicle suddenly jumps. If the accelerator pedal is released/accelerated or decelerated first, and very importantly, in the first gear or in the first "gear", the vehicle stops suddenly;

14) non-inclusion of direction indicators: it is a mistake if the candidates do not mark every change of direction and do not announce the turn to other road users;

15) failing to turn off direction indicators: sometimes candidate/men and women, upon completion of the action with the inclusion of the indicator, fail to turn the indicator off, which may cause different, more times, harmful consequences;

16) watching the wheel/controller: the candidate, especially in the beginning of education, more frequently or for a long time looks at the wheel, which shows that they do not know in which position the lever gear is, which is certainly harmful action and error;

17) keeping the left foot constantly on the clutch pedal while driving: an action that the candidate should not do for at least two reasons: (1) the position of the candidate's foot is uncomfortable, and the clutch blade is unnecessarily consumed more than necessary; (2) the key point for registering an error is that there is no occurrence of successive changes.

Data Acquisition: An important activity to monitor the progress of the ride and determine the type of errors is to collect the data, which has two parts: preparation of recording/collection and recording/collection. In preparing the recording/collection, it was necessary to define the purpose and purpose of the recording, to determine what and how to record it, and to define the use of a particular recorder or list or data collection list. The aim and purpose of determining specific analysis for driving in the examination and analysis of special driving in city traffic and on the training ground 
(with the assumption that it would be given two basic sets should vary and that would like to determine the difference between them). Due to the foregoing reasons, the data is presented on the same type of recording list/list of data collection, but the data in the data recording list are fundamentally different. The goal determines the desire to obtain such information from which one can draw conclusions such as: optimal number of hours of training, the optimal number of hours for teaching unit, for which teaching unit more hours are needed, for which less, etc. A special pattern recording list is prepared, with data of a one single day to monitor the driving of candidates in urban traffic and on the polygon (during training) and a special recording sheet for monitoring the candidates in the exam.

The aforementioned recording/data collection sheet has several introductory headings in the header. It also has two additional types entered, namely:

18) unexpected reactions of the candidate : the candidate may, for any reason, react unexpectedly;

19) sudden reactions from other participants: other participants may, for whatever reason, react unexpectedly.

Data for 15 candidates were entered.

The aforementioned recording/data collection sheet also has several introductory headings. It also has two additional types of errors under numbers 18) and 19), the same as in the first-mentioned recording Journal/Journal for data collection, with the entered data for the three candidates/men and women.

Data for 3 candidates were entered.

Frequency of occurrence of errors types were recorded in both record/data collection lists.

At the bottom recording sheets/lists for data collection are a particularly subscribed frequency: a necessary intervention of the instructor in response to a direct threat due to types of errors candidate/men and women or for types of errors of other traffic (entering the closed crossings, rush to another vehicle, losing direction, etc.). The elements for data collection were:

a) number of candidates: the special records contain all information about the candidate (year of age, recording, will for training, etc.),

b) ordinal number of driving hours of candidates: information to obtain the distribution of corrections or repetitions of types of errors during training,

c) number of kilometers measured: all measured hours of driving are recorded, whether in urban traffic, at the polygon or at the exam. Candidates drove to the polygon on their own,

d) each teaching hour is made by a certain teaching unit, each of which is indicated in the sheet by a special number / code, namely:

III drive to the polygons, the polygon driving on the "front line", driving back and forth, driving the polygon back to Croatian National Theater in Zagreb,

VIII ride to the polygon, left side parking, back ride,

$\mathrm{XIV}$ ride around town,

XVI driving around the city, especially at the crossroads,

XVII uphill and city driving,

XXIX repetition of everything.

The research was mostly done by the author of the graduation paper and instructor Marijan Biščanić, who knew very well the purpose and manner of research and the character of certain types of mistakes. He was partly assisted by fellow instructors from the same profession. The research was quite tedious and exhausting.

Types and additional analysis of collected data: analysis was performed in order to seek some answers about the process of education, and to create conclusions to propose better process of education (partial, but not complete), and some comparisons were made on the basis of references $[15,20,21]$. The analysis is split on the several characteristic areas, namely:

- analysis of the frequency/frequency of the type of errors of individual candidates/hours per driving hour, or indeed per units,

- analysis of the frequency/frequency of the type of mistakes made by individual candidates in polygon driving,

- analysis of the frequency/frequency of types of mistakes made by candidates in the city driving (city driving),

- analysis of the frequency/frequency of the type of candidate's mistakes in the exam.

Error type analysis by driving hours: Hours number: 1, 2, 4, 5, 7, 9. 12. 13. 14, 18, 21 and 22 (12 in total), and candidates number: $4,5,6 \ldots 40$ (37 in total), first of all, a frequency diagram of the type of errors per hour of driving for the candidate no. 4. (in the upper part of Figure 3. coordinate system of abscissa k-hours of driving and ordinates yfrequencies of the type of error), then a diagram of the frequency of the average type of errors per hour of driving for candidate/student no. 4. (in the lower part of Figure 3, the coordinate system of abscissa k- hours of driving and the yfrequency ordinates of the average type of error). Candidate 4 is the candidate with the highest average (41.3\%) types of driving errors, which occurred only in some of the 12 mentioned driving hours, with the amounts: 49, 33, 31, 53, 41 and 33. The second in number is candidate number 8 , with an average number of types of errors of $32.8 \%$, with the frequency of types of errors being: 74, 32, 21, 21 and 28 at certain driving hours. 


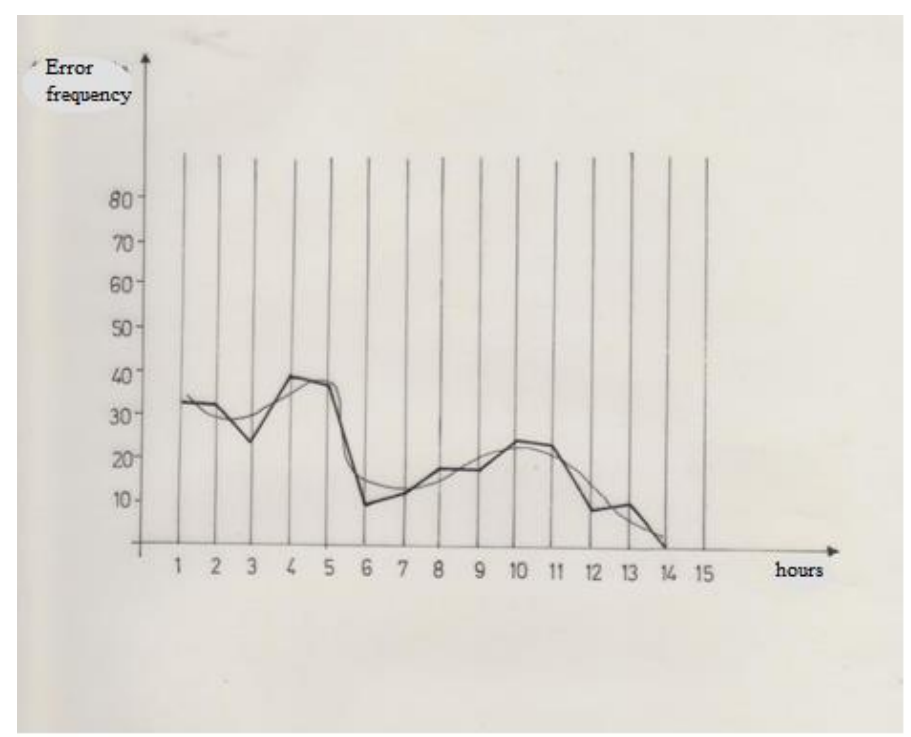

Figure 3. Frequency of types of errors for candidate number 4 Source: Biščanić, M.: Ergonomic Features in the Training of Driving a Car, Graduate Thesis at the Faculty of Mechanical Engineering and Naval Architecture Zagreb, University of Zagreb , Zagreb, 1975, Republic of Croatia

For listed 37 candidates/men and women and 12 above-hour drive is determined, that the average frequency of types of errors in the period from the first to the fifth hour is constant and quite dangerous, then abruptly falls, kind of again begins to increase until the tenth hour and then dropping to fourteenth hour. Frequency of average types of errors for all candidates/maximum for the errors 1, 2, 3, 7 and 11, while the frequency of the other type of error is less. The frequency of error types for all candidates is highest in the first hour, falls by the third hour, then rises by the seventh hour and then falls by the eleventh hour. Analog analysis is performed and for the selected candidates, from which the frequency type defects are largest around six hours, then dropping to the tenth hour, increases to a maximum value in the twentieth and twenty-second hour, and finally, falls again.

A special frequency table for the average types of errors per hour of driving was made for all the candidates observed. This display allows the two analyzes, the analysis of the average kind of errors per candidate and analysis after hours of driving. Analysis by hours of driving each candidate shows that: at the candidate No.1 largest frequency types of errors are in 6,7,20,22, 23 and 24th hour of driving, while the candidates No.2 largest frequency type of errors are in the 4th, 5th, 6th and 17th hour of driving. Further highest frequencies of error types were achieved at: candidate number 4 at the 4th, 5th, 8th and 9th hour of driving; candidate number 5 at the 22nd and 23rd hours of the ride; candidate number 7 in hour 4, 8, 9, 15, 18, 20, 21 and 22; 2 candidates number 9 at the 14th, 20th and 23rd hours; candidate number 12 at 5, 17,18 and 21 hours; candidate number 13 in the 24th hour; candidate number 14 in hours 4, 6, and 8; candidate number 18 at hours 3 and 7; candidate number 21 at hour 8; candidate number 22 at hours 6 and 7. Analysis of the types of errors for all candidates/men and women together after hours of driving shows that the highest frequency types of errors are in the 4th, 5th, 6th, 7th, 8th, 20th, 22nd and 23rd hour drive , etc.

Analysis of the types of errors in driving the polygon (code index: pol): driving on the test site is analyzed separately from driving in the city traffic, in order to do a comparison of these basic sets and determined and random or significant/substantial differences between them. Actions polygonal course units 3 and 8 to online content is total of 34 hours of driving, or a sample of 34 candidates. A summary table of the frequencies of the type of error per candidate and for all 34 candidates is presented in a separate table. The apparent size of certain types of errors in \% for polygon teaching unit 3 and 8 and the results of the accumulation of 34 hours driving from and to are set with results for all candidates together. The average size of individual types of errors in driving the polygon is obtained by dividing the sum of types of errors for each specific type of error with a total of 34 hours of driving on the test site, according to the expression

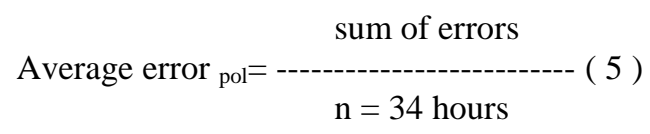

The total sum of the average amounts of all types of errors is $29.73 \%$. Divide the data obtained by each, and the average value of a particular error (for example, type error is the number 4) ), the said amount $29.73 \%$ (the amount 
represents the baseline of $100 \%$ - for calculation of the ratio), average values are obtained of some types of faults in $\%$ for the polygon.

It is noted that the largest average value of individual types of the errors on the training ground, expressed in $\%$, analytically and graphically, are the mistakes number $1,2,3,13,15$, etc. The priority of all errors can be displayed by the Pareto/ABC curve.

Analysis types of errors in city traffic (code index: cd) : The table of all the units of urban driving, thus in addition to the polygonal Units 3 and 8, presents the frequency of low errors from number 1 to 17, for each candidate separately. In particular, the cumulative frequencies of individual types of errors for all candidates and their sum are given, together with the frequencies for all units (except polygon 3 and 8), and those cumulatively amount to 2377 errors.

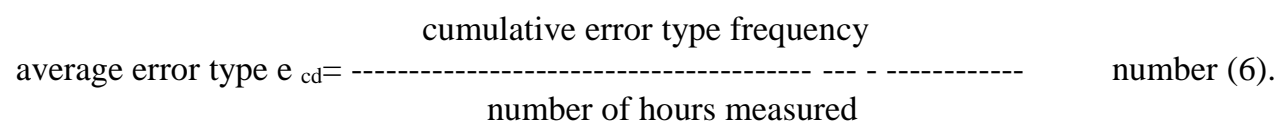

All urban driving units contain a total of 100 driving hours. If the data of the cumulative frequency of the type of error is divided by 100 measured hours, the generalized term first obtained is the average number of types of errors per hour, and then, by listing the data 100 hours, the expression for the specific term is obtained

cumulative frequency types of errors

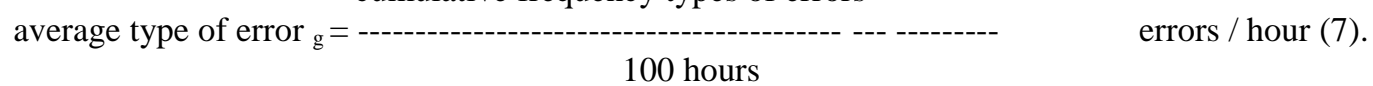

If each average frequency of the type of error per hour is divided by $23.7 \%$ (the total average frequency of all errors is 2377 divided by $100 \%$ ), which represents the base amount of $100 \%$ - to calculate the ratio, the average type of error will be in $\%$. It is noted that for all the candidates in city driving, analytically and graphically, shows that the highest frequency types of errors are number $13,1,16,8,12,3,15,10$, etc. Previous data on cumulative frequency confirm such a conclusion.

Analysis by types of examinations (index code: et): the table shows the frequencies for each of the types of errors 1) to 17), individually for the 7 candidates who took the exam. The columns of the table show numbers showing the distribution of the total frequency of all 7 candidates by each of the 17 types of errors. Lastly, the last column lists the monthly number of driving hours for each candidate. Another table shows the header relationship row-column, that kind of errors 1) to 17) for 7 candidate taking the exam, but it actually represents information median of number of types of errors per hour. Also in the table shows the average frequency of errors for hourly rides for each candidate individually. The table values in one single line are obtained in a way that each data frequency of certain types of defects are divided by the number of hours driving monitored for a candidate. From the above table it shows that for all candidates came out to test the maximum average frequency types of errors 8,13 and 16, while the values of frequencies for other types of errors less. Analysis of the types of errors shows that the highest average frequency type defects history belong: the other error 1, candidates 4, 13 and 22 to error 13 candidates 5, 22 and 13; for the type of error, there are 16 candidates 4, 5 and 22; the error 8 candidates 9, 13 and 22 of the analysis candidates/students revealed that the highest frequency including: candidate 4 toss the type of errors are 1:13; candidate 5 with error types 1 and 13; candidate 9 in error types 1 and 13; candidate 13 for error types 1 and 13; candidate 14 in error types 1 and 13; candidate 21 in error type 1 and 13 ; to candidate 22 the errors 1 and 13.

The conclusion is: the candidate/men and women 21 , who took the test, a small frequency of all kinds of errors in relation to other candidate, whereas for candidates 4 , that is also the position/exam, could not pass because it had a lot of high frequencies for types of errors 1 and 16. Candidate 22, who dropped out, has high frequencies of types of errors $1,13,16$ and 8. All candidates have the highest frequency of types of errors 1 and 13. The instructor must, because of sudden reactions of the candidate, react if a situation of imminent danger arises (vehicle or object crash, red light, loss of direction, etc.,). In measuring the types of candidate errors, the number of times the instructor had to respond was due to a situation of direct danger, namely: due to the types of candidate errors, or because of the types of errors of other road users, where the candidate was not. Collected data were: total 131 hours of measurement clock, type Error 1 to 246 times and occurred Error 2 occurred 58 times. On average, every hour the instructor had to react because of the candidate 246/131= 1.88 times, and because of other participants $58 / 131=0.44$ times. The data indicates that the instructor can expect 1.88 times, exactly 2 times, at every hour to come into danger, from which a timely and quick reaction will save the candidate. Due to other candidates, 0.44 times per hour is in a dangerous situation, that is, virtually every 2 hours, it comes into danger due to other participants. Only drivers, not pedestrians, were measured.

The most important single conclusion of all the above would be:

1) when recording types of errors while driving, only the kind of errors that are made in mastering the action of driving techniques are taken into consideration. Type of error is determined when candidate erroneously performs an action with which was already familiar in detail, 
2) particular attention is paid to the data of the type of errors at the polygon, in urban traffic and at the exam (different basic sets),

3) a total of 19 types of errors are systematically presented and individually defined,

4) in the reference period, all candidates along most repeating the following types of errors: Number 1) (erroneously releases the clutch pedal), Number 2) (engine off/on), Number 3) (clutch pedal insufficiently pressed), Number 7) (gross gear change) and Number 11) (poor bending).

5) All candidates together have the highest number of types of errors for the following driving hours: $22,4,7,6$, $23,8,5$ and 20 ;

6) driving the polygon (Units 3 and 8), all candidates have the highest frequency of the following types of errors: 13) (asynchronized operation of the clutch pedal and the accelerator), 1) (erroneously releases the clutch pedal), 2) (extinguished engine), 3) (the clutch pedal is not pressed enough), 12) (lost in the direction of the shifting), 15) (does not give out direction indicators) and 16) (watching the gearshift while shifting gears),

7) for other teaching units (except 3 and 8), for urban driving, all applicants together have the highest frequencies of the following types of faults: 13) (clutch and throttle pedals not synchronized), 1) (erroneously releases the clutch pedal) , 16) (looks at the gearbox when changing time), 8) (improper gear), 12) (loses direction when changing gears), 3) (clutch pedal not pressed), 15) (does not turn on/off the turn signal) and 10) (losing direction),

8) 7 candidates were followed up to passing the exams: Numbers 4, 5, 9, 13, 14, 21 and 22. Out of the above 7 , candidates who passed were number 4 and 21, Number 22 gave up (did not pass) and did not pass the other 4 candidates.,

9) all 7 observed candidates had the highest frequency of types of errors before the exam, number 1) (incorrectly releasing the clutch pedal) and number 15) (asynchronous work of the clutch and throttle pedal).

10) The instructor comes in a dangerous situation, where he must react timely and properly, because of the candidate 1.88 times per hour, but because of other candidates 0.44 times per hour, or 0.88 times in 2 hours.

\section{ACKNOWLEDGEMENT}

These articles are in memoriam to late BSc.Mech, Eng. Marijan Biščanić (during his graduation thesis, and after that he became a B.Sc.Mech.Eng.on Faculty of Mechanical Engineering and Naval Architecture of Zagreb, University of Zagreb, Republic of Croatia) who is in reference [1], never after and nowhere published, exhibited and prepared rich and worthy theoretical hypothesis of investigation data in personal car driver education, in this, probably, unique in this space and wider, but with special approaches. His work was a very good background for elaborating and processing and editing that subject and belonging content.

We are also grateful to prof. Ph.D. Dragutin Mikšić for several years of collaboration with the last, fourth, author of this article and for using one part of his content about ergonomics from his textbook/manual entitled "Introduction to Ergonomics", reference [2], too.

\section{REFERENCES}

[1] Anohin, P.H.: Philosophical sense/meaning of cybernetic legality. Cybernetic aspects in the investigation of brain work, (english translate); Philosophical sense of cybernetic laws. Cybernetic aspects in the learned work of the brain (russian original), Nauk, Moscow 1970.

[2] Bell, C. (1802): Anatomy of the brain, explained in a series of e-gravings, London, hdl..2027 / nyp: $33433011665720 z ̌$

[3] Đikić, D.: Human Physiology-Selected Topics for the course Human Physiology ..., Faculty of Science, University of Zagreb, Zagreb, 2017/2018.

[4] Elvik, R., Christensen, P., Amundsen, A.: Speed and roads accidents. tOI Reports 740/2004, Institute of Transportation Economics, Oslo, 2004.

[5] Fechnec, G.: Elementer Psychophysik (1860, 2nd ed. 1889)

[6] Gelfand, S.A.: Hearing: An Introduction to Psychological and Physiological Acoustics, Department of Linguistics and Communication Disorders, Queens College of the City University of New York, Flushing New York, Inform Healthcare, New York 2010

[7] Guyton and Hill: Medical Physiology, 12th Edition. Medical edition, Zagreb, 2012

[8] Hatch, F., Maietta, L.: Kinestetics, Infant Handling (on Germany) (2nd durchges, ed.) Bern, Huber, ISBN 9783-456-95987-4

[9] Hergenhahn, B.R., Henley, T.B. (2013): An Introduction to the History of Psychology, 7th Edition 237-238, Cengage Learning

[10] Jacobsen, F.: An Elementary Introduction to Acoustics, Acoustics Technology, Department of Electrical Engineering, Technical University of Denmark, November 2011.

[11] Kandel, E.T., Schwartz, J.H., Jessell, T.M., Siegelbaum, S.A., Hudspeth, A.J.: Principles of Neural Sciences, 5th Edition, McGraw-Hill 2012, ISBN 978-0071398118 
[12] Keros, P., Andreis, I., Gamulin, M.: Anatomy and physiology, Zagreb, 1997.

[13] Korent, D.: Determining the arresting/arresting way/path of motor vehicles for safety traffic function, Faculty of Traffic Sciences, University of Zagreb, Zagreb, 2016.

[14] Kos, J.: Injuries and Wounds. definition, etiology and division, Wound healing and general principles of treatment, Faculty of Veterinary Medicine in Zagreb, Department of Surgery, Orthopedics and Ophthalmology, University of Zagreb, Zagreb, 2008.

[15] Law on Road Traffic Safety; OG 67/2008, 48/10, 74/11, 80/13, 158/13, 92/14, 64/15, Zagreb, 2015.

[16] Marble, M.F. (February 2016): Vision, Eye Disease, and Art 2015, Keeler Lecture, Eye (30) 2 287-303, doi:10.138/Eye.205.197, PMC: 4763116, PMID 26563659

[17] Metikos, D., Prot, F., Horvat, V., Kuleš, B., Hofman, E. : Basic motor skills for examining/subject with

exceendingly motoric properties/attributes, Faculty of Physical Education, University of Zagreb, Kinesiology, Vol 1281982 / orig. no. pp 21-62

[18] PC portal Actually.hr (2014): These are the types of most stupid/foolish drivers who every day we meet/find/ happen in traffic. Are you among them?

[19] Piri, G.: The Relationship of Intelligence with Success in Passing Driving Exams and the Number of Traffic Offenses and Accidents, Philosophically, University of Zagreb, Zagreb, 2007.

[20] Driving Exam Program: NN / 155, 108/2009, 191/2011, 146/2012, Zagreb, 2012.

[21] Rulebook / Regulations / Rules / Bylaw on training / qualifications for driver candidates - instructor for diving NN/12, www.instructor-driving.com.hr/rulebook-about-training-driving, NN 124/2912, 129/2022, 146/ 2012,151 / 2013, 160/2013, Zagreb, 2013.

[22] Sokac, T.: Effects of Training Process of Candidates for Drivers on Traffic Safety, Graduate, Faculty of Traffic Sciences, University of Zagreb, September 2016.

[23] Stevens, SS, Davis, H. (196): Hearing, its Psychology and Psychology, Wiley

[25] Šverko, B.: (2006): Psychology, Zagreb, School Book

[26] Turkalj, K.: Improvement of the Driver Candidate Education System as a Function of Increasing Security of Road Traffic, Graduate, Zagreb, 2016.

[27] Whitfield, TWA, Wiltshire, TJ (1990): Color Psychology, A Critical Review, Genetic, Social and Genetic Psychology, 16 (4, 387-392)

[28] Zarevski, P., Havelka, M. (2014): Psychology, School Book

[29] Car, M., Hađina, J., Car, M., Biščanić, M.: Ergonomics and Ergonomic Analysis of Driving Education System by Personal Car with Supporting IT, Asian Journal of Computer and Information Science (ISSN: 2321-5658), Volume 06-Issue 02, October 2019. 